\title{
Atrial Fibrillation: Current Perspective
}

\author{
R Ahmed ${ }^{1}$, DJ Schamp ${ }^{2}$ \\ ${ }^{1}$ Cardiac Electrophysiologist, Midatlantic Cardiovascular Associates, Clinical Assistant Professor of \\ Medicine, University of Maryland School of Medicine, Baltimore, USA, ${ }^{2}$ Cardiac Electrophysiologist, \\ Midatlantic Cardiovascular Associates, Baltimore, USA
}

(Cardiovasc. j. 2009; 1(2) : 148-155)

\section{Introduction and Epidemiology:}

Atrial fibrillation is the commonest cardiac arrhythmia encountered in adult population. Dr. McKenzie initially described Atrial fibrillation in 1902. In one of his patients with mitral stenosis and loud pre-systolic murmur, he found that the appearance of irregularity of the pulse coincided with the disappearance of the pre-systolic murmur and "a" wave in jugular venous tracing. He called this "paralysis of the auricle". Thomas Lewis in 1910 first used the term "auricular fibrillation". ${ }^{1}$

Estimated prevalence of atrial fibrillation in United Sates is about 1 to $6 \%$ and at any given time it affects about 2.3 million people. ${ }^{2,3}$ The prevalence of atrial fibrillation rises sharply with age and exceeds $5 \%$ in subjects over the age of 70 . Atrial fibrillation is more prevalent in men than women. The annual incidence of atrial fibrillation is about $0.3 \%$ in men and $0.2 \%$ in women between the ages 55 and 64 . Over the age of 85 the annual incidence of atrial fibrillation is $3.8 \%$ in men and $3.2 \%$ in women. ${ }^{4,5}$

\section{Types of Atrial Fibrillation:}

Traditionally atrial fibrillation has been classified as Paroxysmal and Chronic. Paroxysmal atrial fibrillation was defined as episodes of atrial fibrillation lasting short period of time and converting spontaneously back to sinus rhythm. A newer and more practical classification has divided atrial fibrillation into the following groups. ${ }^{6}$

1. Intermittent atrial fibrillation is defined as episodes of atrial fibrillation terminating spontaneously into sinus rhythm and lasting less than 7 days. These episodes can be recurrent.

2. Persistent atrial fibrillation is an episode of atrial fibrillation that lasts more than 7 days and no attempt has ever been made to cardiovert to sinus rhythm.
3. Permanent atrial fibrillation is the term used when an episode has lasted more than one year and has failed cardioversion and medical management.

\section{Etiology and Pathophysiology of Atrial Fibrillation:}

Atrial fibrillation is associated with many different cardiac and non-cardiac disorders. In about $2 \%$ of patients with atrial fibrillation there are no identifiable associated cardiac or non-cardiac disorders. These are called "Lone atrial fibrillation". Majority of the patients with lone atrial fibrillation has paroxysmal atrial fibrillation and they are relatively younger with excellent longterm prognosis. ${ }^{7}, 8$.

Age related atrial fibrosis is probably an important cause of atrial fibrillation. Any condition that leads to distension, increased pressure or hypertrophy of the atria can cause atrial fibrillation. These conditions are mitral valve disease, ventricular hypertrophy from any cause especially hypertension, myocardial disease leading to systolic or diastolic dysfunction and pulmonary disease. Other causes of atrial fibrillation are atrial ischemia, inflammation or infiltrative disease of the atria (pericarditis, myocarditis, amyloidosis), increased sympathetic activity (hyperthyroidism, pheochromocytoma, exercise, caffeine intake, sympathomimetic drugs, pulmonary embolism, post cardiac surgery), intoxicant such as alcohol, increased parasympathetic activity such as during sleep, cancer in or adjacent to atrial wall, sick sinus syndrome and Wolf Parkinson White syndrome. A rare variety of familial or genetic atrial fibrillation has also been identified. ${ }^{9}$

There is no pathologic change in the atrium that is specific for atrial fibrillation. With aging there is increase in fatty tissue in the atrium. This change and also fibrosis, infiltration and

Address Correspondence : Rafique Ahmed, Cardiac Electrophysiologist, Midatlantic Cardiovascular Associates, Clinical Assistant Professor of Medicine, University of Maryland School of Medicine, Baltimore, USA, 
inflammation are the changes that are associated with atrial fibrillation. These changes can also be precipitated by persistence of atrial fibrillation. There is no gross pathological change of the atrium inpatients with lone atrial fibrillation.

Knowledge of electrophysiological changes in the atrium inpatients with atrial fibrillation has expanded over the last decade. The atrial action potential duration and effective refractory period of atrial tissue is shortened in atrial fibrillation. ${ }^{10}$ In addition atrial tissue looses the property of rate dependent change in action potential. Experimental and clinical studies suggest that atrial fibrillation can be initiated and sustained by a single source or multiple source electrophysiological abnormality. Single source can be an automatic focus, a single stable fixed macrore-entrant circuit with short cycle length or a single stable macro-reentrant circuit drifting through the atria. Multiple source atrial fibrillation can be from multiple automatic foci, multiple re-entrant circuits in the atria or multiple small wavelets wandering simultaneously around the atria. ${ }^{11}$ Autonomic nervous system, especially the vagus nerves plays an important role in the initiation and maintenance of atrial fibrillation. With vagal stimulation there is shortening of atrial refractory period and increase in the spatial dispersion of refractoriness. When a premature impulse encounters such an area the wavefront is blocked and it travels in other directions. When the time required for the impulse to travel around the block exceeds the refractory period of the cells proximal to the block, these cells will be re-excited and reentrant arrhythmia can be initiated. ${ }^{12}$

\section{Diagnosis:}

Atrial fibrillation can be easily recognized on the 12 lead electrocardiogram. In atrial fibrillation the $\mathrm{P}$ waves are replaced by fibrillation waveforms that occur at the rate faster than 350 beats per minute. The fibrillation waves are best visible in leads V1 and V2. The ventricular rate depends on $\mathrm{AV}$ conduction and the RR intervals are irregularly irregular.

\section{Clinical Consequences of atrial fibrillation:}

Most important and devastating clinical consequence of atrial fibrillation is embolic stroke. The yearly risk of stroke in atrial fibrillation is about $1.5 \%$ between ages 50 to $59,2.8 \%$ between ages 60 to69, $9.9 \%$ between ages 70 to 79 and $23.5 \%$ between ages 80 to 89 years. ${ }^{13}$ A report from investigators of five large randomized trial of anticoagulation in atrial fibrillation has identified patients at highest risk of stroke. ${ }^{14}$ They found that the risk of stroke in atrial fibrillation was about $12 \%$ per year in patients with prior history of stroke or TIA, 8 to $9 \%$ in diabetics, 5 to $6 \%$ in hypertension and 6 to $7 \%$ per year in patients with heart failure. Prior history of stroke / TIA, hypertension, poor LV function, age $>75$ years, rheumatic mitral valve diseases and presence of prosthetic heart valves are considered high risk factors for development of stroke in atrial fibrillation. Ages 65 to 75 years, diabetes and coronary artery disease are considered moderate risk factors for stroke in atrial fibrillation. ${ }^{15,} 16$ The risk of stroke is similar in intermittent or permanent atrial fibrillation. ${ }^{17}$

Patients with atrial fibrillation have increased mortality compared to similar population without atrial fibrillation. In Framingham study atrial fibrillation was associated with 1.5 times increased risk of death in men and 1.9 times in women. In addition atrial fibrillation can cause significant impairment of quality of life with symptoms such as palpitation, dyspnoea, chest pain, dizziness, syncope, fatigue. Atrial fibrillation with rapid ventricular response can precipitate heart failure; angina and can also lead to the development of tachycardia-induced cardiomyopathy.

\section{Initial Evaluation:}

When a patient presents with apparent atrial fibrillation a careful history should be taken and physical examination performed. Diagnosis of atrial fibrillation should be confirmed 12 lead ECG. If the patient is hemodynamically unstable then emergency electrical cardioversion should be performed. Patient should be evaluated for any associated complications such as heart failure, stroke or any other thromboembolic complications.

The following tests should be done or considered as appropriate in all new patients. Patients should have full blood count, prothrombin time and partial thromboplastin time, serum electrolytes, blood urea nitrogen and creatinine. Thyroid function tests to rule out thyrotoxicosis should be done on all new patients. 24-hour Holter monitor is helpful in the diagnosis of paroxysmal atrial fibrillation. 
A chest x-ray: it may provide clue to congenital heart disease or provide information regarding heart failure and size of the heart. Echocardiography is an important investigation to assess valvular structure and to assess left ventricular function. Trans-esophageal echocardiography may be needed in selected patients, especially if there is any plan of cardioversion urgently. Exercise stress test should only be considered if there is any suspicion of coronary disease.

\section{Echocardiography in atrial fibrillation:}

Echocardiography is an important tool in the assessment and risk stratification of patients with atrial fibrillation. It gives information regarding structural heart disease including valvular heart disease, left ventricular function, left atrial size, presence of clot in the left atrium and left atrial smoke (echogenic shadow near the wall which has been shown to increase risk of thromboembolism). Left ventricular dysfunction (relative risk 2.6) and dilated left atrium (left atrium $>5.7 \mathrm{~cm}$, relative risk 2.7) are independent risk predictors of stroke in atrial fibrillation. ${ }^{18}$

Stroke occurs mainly from clots in the left atrial appendage, which is sometimes poorly visualized by transthoracic echocardiography. Transesophageal echocardiography is more sensitive (92\%) and specific (98\%) in detecting left atrial clot. ${ }^{19}$ There is emerging data to suggest that absence of atrial clot as seen by transesophageal echocardiography virtually eliminates the risk of thromboembolic event. ${ }^{20} \mathrm{At}$ the present time it is wise and cost effective to restrict the use of transesophageal echocardiography in situations where patients present with atrial fibrillation of $>48$ hours duration and acute cardioversion is a high priority (acute heart failure, angina, rapid ventricular response with low blood pressure etc). The absence of left atrial clot does not obviate the need for anticoagulation after cardioversion, since post cardioversion atrial stunning may still occur. ${ }^{21}$

\section{Pharmacological approaches to management of atrial fibrillation:}

1. Control of ventricular rate

2. Anticoagulation for prevention of thromboembolic events.
3. Restoration of sinus rhythm - electrical or chemical cardioversion.

4. Maintenance of sinus rhythm with antiarrhythmic drugs.

\section{Control of ventricular rate:}

The first step in the management of patients with atrial fibrillation is to slow down the ventricular rate. Drugs used in controlling ventricular rate are digoxin, beta-blockers and calcium channel blockers.

Digoxin: This was the first drug that was used to control ventricular rate in atrial fibrillation. The limitations of digoxin are long time to onset of action and reversal of its effect by catecholamines. ${ }^{22}$ As a monotherapy it is usually sufficient for elderly or less active patients. In more active subjects it is a very effective drug in combination with beta-blockers and calcium channel blockers. Its main advantage is that it can be used in patients with very low ejection fraction. Digoxin has no role in converting patients to sinus rhythm. ${ }^{23,} 24$

Beta-blockers and calcium channel blockers alone or in combination with digoxin are effective in controlling ventricular rate. ${ }^{25} \mathrm{~A}$ recent study showed that combination of digoxin and betablocker was most effective in the control of resting, exercise and 24 hour heart rate. ${ }^{26}$ The use of calcium channel blockers are limited by the fact that they can depress left ventricular function and may not be tolerated by patients who have poor left ventricular function.

The AV node blocking drugs should not be used in the setting of Wolf Parkinson White syndrome with atrial fibrillation and antegrade conduction through the accessory pathway. In such a patient intravenous procainamide is the drug of choice.

Intravenous drugs should be considered when rapid slowing of ventricular rate is desirable. This includes patients with congestive heart failure, chest pain, uncontrolled and rapid ventricular response and WPW syndrome. Metoprolol can be given intravenously at a dose of $5 \mathrm{mg}$ every 2 to 5 minutes, up to a maximum dose of $15 \mathrm{mg}$. Diltiazem is usually given at a bolus of $0.25 \mathrm{mg} /$ $\mathrm{kg}$, followed by infusion rate of 10-15 mg/hour. 


\section{Goals of heart rate control:}

1. Resting heart rate is $\leq 80 \mathrm{bpm}$ (apical pulse or by ECG).

2. 24 hour average heart rate $<80 \mathrm{bpm}$ with no single hour average heart rate more than 100 bpm by Holter monitor.

3. Exercise heart rate not exceeding $85 \%$ of age predicted heart rate with low-level exercise (Bruce stage I).

\section{Anticoagulation in atrial fibrillation:}

Multiple randomized studies have established the benefit of anticoagulation and or aspirin in reducing risk of stroke in atrial fibrillation $(27,28,29,30)$. Patients with atrial fibrillation who are at high risk of stroke be considered for anticoagulation with warfarin and or aspirin. Prior history of stroke or TIA, systemic embolism, hypertension, poor LV function, age more than 75 years, rheumatic mitral valve disease and presence of prosthetic heart valves are considered as factors associated with high risk of stroke. Two have looked at the risk of stroke in different cohorts of non-valvular atrial fibrillation and have developed CHADS scoring system to assess risk of stroke and need for anticoagulation. ${ }^{31,32}$

\section{CHADS score and risk of stroke:}

\begin{tabular}{lc}
\hline Risk factors & Score \\
\hline Congestive Heart Failure & 1 \\
Hypertension & 1 \\
Age 75 or older & 1 \\
Diabetes & 1 \\
Stroke, TIA history & 2 \\
\hline
\end{tabular}

\begin{tabular}{cc}
\hline CHADS Score & Yearly Risk of Stroke \\
\hline 0 & $1.9 \%$ \\
1 & $2.8 \%$ \\
2 & $4.0 \%$ \\
3 & $5.9 \%$ \\
4 & $8.5 \%$ \\
5 & $12.5 \%$ \\
6 & $18.2 \%$ \\
\hline
\end{tabular}

The current recommendations for anticoagulation in patients with non-valvular atrial fibrillation based on CHADS score is as follow:

$\begin{array}{ll}\text { CHADS score } 0 & \text { - Aspirin } \\ \text { CHADS score } 1 & \text { - Aspirin or Warfarin } \\ \text { CHADS score } 2 \text { or more } & \text { - Warfarin }\end{array}$

One of the controversial issues in anticoagulation is its duration. Traditional approach was anticoagulation for 4 weeks prior to cardioversion and then to discontinue anticoagulation after 4 weeks if sinus rhythm was maintained. Results of the AFFIRM and RACE trial found that stroke was higher when warfarin was discontinued in the sinus rhythm arm of the studies. ${ }^{33,} 34$ The reason for this can be episodes of asymptomatic atrial fibrillation or may be these patients are inherently at higher risk of stroke from aortic plaque formation. It is now a felt that these patients should be anticoagulated indefinitely with warfarin.

Patients on oral anticoagulation with warfarin should have blood checked regularly to maintain INR (International Normalized Ratio) within therapeutic range that is 2 to 3 for most patients with atrial fibrillation. INR should be kept between 2.5 to 3.5 in patients with rheumatic valve disease, prosthetic heart valves, prior history stroke/TIA and in the presence of atrial thrombus. Anticoagulation is usually initiated at a dose of $5 \mathrm{mg}$ daily. Blood should be checked every week until therapeutic INR has been achieved and stabilized. After that INR should be checked monthly. Maintenance of therapeutic INR will give maximum benefit of warfarin while minimizing risk of bleeding.

One of the difficulties of warfarin use is requirement of regular blood test to maintain therapeutic INR. This has led to the search to develop oral agents. Direct thrombin inhibitors have been found to be promising. Ximegalatran is an oral direct thrombin inhibitor and has undergone large randomized trial in patients with atrial fibrillation. The SPORTIF V study compared ximegalatran with warfarin in patients with non-valvular atrial fibrillation and ximegalatran was found to be as effective as warfarin in reducing risk of stroke. ${ }^{35}$ This drug was not market released because some concern about impairment of liver function. Second generation of direct thrombin inhibitors are in development and hopefully will be available for use in future.

\section{Restoration of sinus rhythm:}

Traditional reasons for restoring sinus rhythm in patients with atrial fibrillation were appropriate 
and physiologic rate control, regularization of heart rhythm, restoration of atrial contribution to cardiac output with improvement in hemodynamics, prevention of left atrial dilatation, prevention of left ventricular dysfunction, relief of symptoms, improved quality of life and reduction of thromboembolic complications. The finding of studies on rate and rhythm control has challenged these reasons.

About $40-50 \%$ of patients with atrial fibrillation less than 1-week duration will convert spontaneously to sinus rhythm with in 24 hours without antiarrhythmic therapy. ${ }^{8,9}$

Cardioversion should be considered in every patient presenting with atrial fibrillation for the first time. Atrial fibrillation of $>1$ year and left atrial size $>45$ $\mathrm{mm}$ is associated with high recurrence rate, 36,37 but this should not prevent one from cardioverting the patients.

\section{Methods of cardioversion:}

1. Electrical cardioversion: Success rate of electrical cardioversion for new patients is in excess of $90 \%$. ${ }^{36,38}$ Cardioversion should be started with 200J synchronized direct current shock; if that fails then two further shocks at 360J should be given. Newer biphasic defibrillators have higher efficacy and requires less energy. It is very important to perform electrical cardioversion under light general anesthesia. Many of these patients will require repeat cardioversion and the role of a painless procedure cannot be over emphasized.

\section{Pharmacological Cardioversion:}

i) Intravenous Ibutilide: This is the only drug approved in USA for acute cardioversion of atrial flutter/fibrillation. The dose is $1 \mathrm{mg}$ given over 10 minutes, which may be repeated 10 minutes later. The conversion rate in atrial fibrillation is $45 \%$ with $5.1 \%$ incidence of torsade de pointes. ${ }^{39}$ This drug should be reserved for patients who refuse electrical cardioversion or is not a candidate for anesthesia.

ii) Oral antiarrhythmics: Class $1 \mathrm{C}$ agents Flecainide and propafenone have a success rate of $70-90 \%$ in converting atrial fibrillation in patients without structural heart disease. ${ }^{40}$, 41 These drugs are not approved for such use in USA. Other oral agents such as amiodarone, quinidine has lower success rate in converting patients to sinus rhythm.

Rate control versus maintenance of sinus rhythm in Atrial Fibrillation:

Five different randomized studies have compared rate control versus maintenance of sinus rhythm inpatients with atrial fibrillation. $33,34,42,43,44$ The largest study was AFFIRM trial that enrolled 4060 patients with a follow up of 3.5 years. All these studies showed that quality of life and survival was similar in both groups.

\section{Maintenance of Sinus rhythm:}

Without antiarrhythmic drug therapy about 75\% of patients will have recurrent atrial fibrillation. ${ }^{45}$ The risk of recurrence is dependent on underlying LV function, duration of atrial fibrillation, left atrial size and age of the patient. ${ }^{36,37,46}$ Antiarrhythmic drugs are able to prevent recurrences of atrial fibrillation with varying degree of success.

Drugs used for maintenance of sinus rhythm are class IA agents quinidine, procainamide, disopyramide, class IC agents flecainide, propafenone and class III agents Amiodarone, sotalol, Dofetilide. In patients without any structural heart disease Class IC agent flecainide is a very effective medicine in maintaining sinus rhythm. Class I agents are known to increase mortality in patients with structural heart disease especially coronary artery disease and are avoided in such patients. ${ }^{47}$ Amiodarone is the most effective antiarrhythmic agent available to us for maintenance of sinus rhythm. However amiodarone has multiple systemic and irreversible side effects and is now used for older patients also patients with structural heart disease who are strong candidates for restoration of sinus rhythm. Sotalol is not as effective as amiodarone but has no long-term side effects and is being used more in patients with atrial fibrillation. A new drug that is similar to amiodarone without its side effects is being developed.

The most realistic measure of efficacy of antiarrhythmic therapy is the frequency of recurrence not recurrence per se. It is acceptable to continue antiarrhythmic therapy if patient has infrequent recurrences. 
In view of the findings of the rate and rhythm control studies the use of antiarrhythmic drugs has changed significantly. Many physicians now will reserve antiarrhythmic drug therapy for atrial fibrillation with hemodynamic compromise or frequent symptomatic recurrences with rapid ventricular response. Newly diagnosed atrial fibrillation of unknown duration should be cardioverted after proper anticoagulation without antiarrhythmics. Antiarrhythmics in this group should be used if there is recurrence after first cardioversion.

The choice and sequence of antiarrhythmic drug usages varies from physician to physician. In patients with lone atrial fibrillation (no structural heart disease) drug of first choice in our practice is either flecainide or propafenone. If flecainide/ propafenone fails then we consider sotalol. In the past if sotalol failed we used to consider using amiodarone. But now we either consider rate control or refer patients for curative procedure such as radio frequency catheter ablation. In patients with structural heart disease amiodarone is the drug of choice if patients have low ejection fraction. Sotalol can be used in this group if LV ejection fraction is more than $40 \%$.

There is no standard guideline as to the number of recurrences of atrial fibrillation that is acceptable while patients are on antiarrhythmic regimen. Possibly three recurrences per year requiring cardioversion is an acceptable number. However, based on the rate versus rhythm control studies it is not unreasonable to follow rate control strategy when there are multiple recurrences of atrial fibrillation.

\section{Non-Pharmacological management of atrial fibrillation:}

1. AV node ablation with permanent pacemaker implantation: This is reserved for patients with atrial fibrillation and rapid ventricular response despite maximal medical management. However, prior to proceeding with this procedure patient should be evaluated for atrial fibrillation ablation. If they have failed atrial fibrillation ablation or if they are not candidate for atrial fibrillation ablation then it is reasonable to perform AV node ablation followed by permanent pacemaker insertion.
2. Radiofrequency ablation of atrial fibrillation: This is evolving as a new tool in the treatment of atrial fibrillation. This has the potential to cure atrial fibrillation. There are multiple methodologies in use, but most share the electrical isolation of atrial fibrillation triggers which arise predominantly from the pulmonary veins. Circumferential isolation of the pulmonary veins is often combined with ablation of atrial vagal ganglia. Success rate of this procedure in restoring sinus rhythm is about 60 to $70 \%$. Preliminary reports suggest a recurrence rate of up to $50 \%$ over a period of about 2 years. The procedure time is still very long and varies from 5 to 10 hours and the risk of major complication is in the range of 5 to $7 \%$ including risk of stroke, pulmonary vein stenosis and atrial rupture. At this time this procedure should be reserved for carefully selected highly symptomatic patients who have failed all other medical options.

3. Surgical Maze procedure: This is a very invasive procedure requiring sternotomy. In this procedure the atria are cut into pieces and sutured back together thereby preventing the maintenance of the reentrant circuits required for atrial fibrillation. Success rate of this procedure in maintaining sinus rhythm is more than $90 \%$. Most of the time this procedure is done concomitant to other cardiac surgical procedures. There are modified versions of this procedure using minithoracotomy or thoracoscopic approaches with use of either cryo or radiofrequency ablation to isolate the pulmonary veins and ablate the parasympathetic ganglia. Initial results are promising, but long-term followup data will be required to evaluate this procedure further.

\section{References:}

1. Sir James Mackenzie: Principles of diagnosis and treatment of heart affections. $4^{\text {th }}$ edition, Oxford Univ Press, London, 1918

2. Go AS, Hylek EM, Phillips KA, et al. Prevalence of diagnosed atrial fibrillation in adults: National implications for the rhythm management and stroke prevention: the anticoagulation and risk factors in atrial fibrillation (ATRIA) study. JAMA 2001; 285:2370-2375

3. Feinberg WM, Blackshear JL, Laupacis A, et al. Prevalence, age distribution and gender of patients with atrial fibrillation: analysis and implications. Arch Internal Med 1995; 155:469-473 
4. Benjamin EJ, Levy D, Vaziri SM et al. Independent risk factors for atrial fibrillation in a population based cohort: the Framingham Heart Study. JAMA 1994; 271:840844

5. Psaty BM, Manolio TA, Kuller LH, et al. Incidence of and risk factors for atrial fibrillation in older adults. Circulation 1997; 96:2455-2461

6. Fuster V; Ryden LE; Cannom DS; Crijns HJ, et al. ACC/ AHA/ESC 2006 Guidelines for the Management of Patients With Atrial Fibrillation A Report of the American College of Cardiology/American Heart Association Task Force on Practice Guidelines and the European Society of Cardiology Committee for Practice Guidelines (Writing Committee to Revise the 2001 Guidelines) J Am Coll Cardiol. 2006; 15:48(4): e149246

7. Brand FN, Abbott RD, Kannel WB, Wolf PA. Characteristics and prognosis of lone atrial fibrillation: 30-year follow-up in the Framingham Study. JAMA 1985; 254:3449-3453.

8. Kopecky SL, Gersh BJ, McGoon MD et al: The natural history of lone atrial fibrillation: A population-based study over three decades. N Eng J Med 1987; 317:669674

9. Podrid PJ: Etiology and pathogenesis of atrial fibrillation. In Kowey P, Nacarelli GV, eds: Atrial Fibrillation. Merkel Dekker, NY, 2005, p 27-60

10. Le Heuzy JY, Boutjdir M, Gagey S et al. Cellular aspects of atrial vulnerability. In Attuel P, Coumel P, Janse MJ, eds: Atrium in Health and Disease. Futura, Mt Kisco, NY, 1989;81-94

11. Blaauw Y, Schotten U and Allessie MA. Preclinical models: What clinical lesson have we learned. In Kowey P, Nacarelli GV, eds: Atrial Fibrillation. Merkel Dekker, NY, 2005, 83-108

12. Allesi R, Nusynowitz M, Abildskov J, Moe G. Nonuniform distribution of vagal effects on the atrial refractory period. AM J Physiol 1958; 194:406-410

13. Wolf PA, Abbott Rd, Kannel WB. Atrial Fibrillation as an independent risk factor for stroke: The Framingham Study. Stroke 1991; 22:983-988

14. Atrial Fibrillation Investigators. Risk factors fro stroke and efficacy of antithrombotic therapy in atrial fibrillation. Arch Intern Med 1994; 154:1449-1457

15. Albers GW, Dalen JE, Laupacis A, et al. Antithrombotic therapy in atrial fibrillation. Chest 2001; 119:194S-206S.

16. ACC/AHA/ESC guidelines for the management of patients with atrial fibrillation: executive summary. $J$ Am Coll Cardiol 2001; 38:1231-1265

17. Hart RG; Pearce LA; Rothbart RM; McAnulty JH; Asinger RW; Halperin JL. Stroke with intermittent atrial fibrillation: incidence and predictors during aspirin therapy. Stroke Prevention in Atrial Fibrillation Investigators. $\mathrm{J} \mathrm{Am} \mathrm{Coll}$ Cardiol 2000 Jan; 35(1): 183-187.
18. Stroke Prevention in Atrial Fibrillation Investigators. Predictors of thromboembolism in atrial fibrillation II: echocardiographic features of patients at risk. Ann Intern Med. 1992; 116:6-12

19. Manning WJ, Silverman DI, Gordon SP, Kromholz HM, Douglas PS. Cardioversion from atrial fibrillation without prolonged anticoagulation with use of transesophageal echocardiography to exclude the presence of atrial thrombi. N Engl J Med. 1993; 323:750-755

20. Falcone RA, Morady F, Armstrong WF. Transesophageal echocardiograpic evaluation of left atrial appendage function and spontaneous contrast formation after chemical and electrical cardioversion of atrial fibrillation. Am J Cardiol. 1996; 78:435-439

21. Hata JS, Aryres RW, Biller J, Adams HP, Stuhlmuller J, Burns T, Kerber R, Vanderberg B. Impact of transesophageal echocardiography on anticoagulation management of patients admitted with focal cerebral ischemia. Am J Cardiol 1993;72:707-710

22. Falk RH, Lesvitt JI. Digoxin for atrial fibrillation: a drug whose time has gone? Ann Intern Med 1991; 114:573-575

23. Jordeans L, Trouerbach J, Calle P, et al. Conversion of atrial fibrillation to sinus rhythm and rate control by digoxin in comparison to placebo. Eur Heart J 1997; 18:643-648

24. The Digitalis in Acute Atrial Fibrillation (DAAF) Trial Group. Intravenous digoxin in acute atrial fibrillation. Result of a randomized, placebo controlled multicenter trial in 239 patients. Eur Heart $J$ 1997; 18:649-654

25. Falk RH. Pharmacologic control of the ventricular rate in atrial fibrillation. In Falk RH, Podrid PJ eds. Atrial Fibrillation: mechanisms and management. LippincottRaven Publishers, Philadelphia, 1997; 299-327

26. Farshi R, Kistner D, Sarma JSM, et al. Ventricular rate control in chronic atrial fibrillation during daily activity and programmed exercise: a crossover open-label study of five drug regimen. J AM Coll Cardiol 199; 33:304-310

27. Petersen P, Boysen G, Godtfredsen J, et al for the Copenhagen AFASAK Study. Placebo controlled, randomized trial of warfarin and aspirin for the prevention of thromboembolic complication s in chronic atrial fibrillation. Lancet 1989; 1:175-179.

28. Stroke Preventionin Atrial Fibrillation Investigators: Stroke prevention in atrial fibrillation study: final results. Circulation 1991; 84:527-539.

29. Stroke Preventionin Atrial Fibrillation Investigators: Warfarin versus asprin for the prevention of thromboembolism in atrial fibrillation: Stroke prevention in atrial fibrillation II study. Lancet 1994; 343:687-691.

30. Hart RG, Benavente O, McBride R, et al. Antithrombotic therapy to prevent stroke in patients with atrial fibrillation: a meta-analysis. Arch intern Med 1999; 131:492-501 
31. Gage BF, van Walraven C, Pearce L, et al. Selecting patients with atrial fibrillation for anticoagulation: stroke risk stratification in patients taking aspirin. Circulation 2004; 110(16): 2287-92

32. Gage BF, Waterman AD, Shannon W, et al. Validation of clinical classification schemes for predicting stroke: results for-national registry of atrial fibrillation. JAMA 2001; 285(22): 2864-70

33. Wyse DG; Waldo AL; DiMarco JP; et al. A comparison of rate control and rhythm control in patients with atrial fibrillation. N Engl J Med 2002, 5; 347(23): 1825-33.

34. Van Gelder IC; Hagens VE; Bosker HA et al; A comparison of rate control and rhythm control in patients with recurrent persistent atrial fibrillation. $N$ Engl $J$ Med 2002, 5; 347(23): 1834-40.

35. Sportif Executive Committee for the Sportif V Investigators. Ximegalatran vs Warfarin for Stroke Prevention in patients with non-valvular atrial fibrillation. JAMA 2005; 293:690-698

36. Morris JJJ, Peter RH, McIntosh HD. Electrical cardioversion of atrial fibrillation: immediate and longterm results and selection of patients. Ann Intern Med. $1966 ; 65: 216-231$

37. Dethy M, Chassat C, Roy D, Mercier LA. Doppler echocardiographic predictors of recurrence of atrial fibrillation after cardioversion. Am J Cardiol. 1988; 62:723-726

38. Dalzell GW, Anderson J, Adgey AA. Factors determining success and energy requirements for cardioversion of atrial fibrillation. Q J Med 1990; 82:903913

39. Ellenbogen KA, Stambler BS, Wood MA et al. For the Ibutilide investigators. Efficacy of intravenous ibutilide for rapid termination of atrial fibrillation or flutter to sinus rhythm: A dose response study. J Am Coll Cardiol 1996; 28:130-136
40. Capucci A, Lenzi T, Boraiani G, et al. Effectiveness of loading oral flecainide for converting recent onset atrial fibrillation to sinus rhythm in patients without organic heart disease or with only systemic hypertension. Am J Cardiol 1992; 70:69-72

41. Suttorp MJ, Kingma JH, Jealous ER, et al. The value of class $1 \mathrm{C}$ antiarrhythmic drugs for acute conversion of paroxysmal atrial fibrillation or flutter to sinus rhythm. J Am Coll Cardiol 1990; 16:1722-1727.

42. Hohnloser SH, Kuck KH, Lilienthal J for PIAF Investigatorr: Rhythm or rate control in atrial fibrillation - Pharmacologic Intervention in Atrial Fibrillation (PIAF): a randomized trial. Lancet 2000; 356:1789-1794,

43. Brignole M, Menozzi C, Gasparini M, et al for the PAF2 investigators: An evaluation of the strategy of maintenance of sinus rhythm by antiarrhythmic drug therapy after ablation and pacing therapy in patients with paroxysmal atrial fibrillation. Eur Heart $J$ 2002; 23:892-900,

44. Carlsson L, Miketic S, Windeler J, et al for the STAF investigators: Ransdomized trial of rate control versus rhythm control in persistent atrial fibrillation: the Strategies of Treattment of Atrial Fibrillation (STAF) trial. J AM Coll Cardiol 2003; 41:1690-2375

45. Coplen SE, Antman EM, Berlin JA, et al. Efficacy and safety of quinidine therapy in maintenance of sinus rhythm after cardioversion: A meta analysis of randomized control trials. Circulation 1990; 82:11061116

46. Vaziri SM, Larson MG, Benjamin EJ, Levy D. Echocardiographic predictors of non-rheumatic atrial fibrillation. The Framingham Heart Study. Circulation 1994; 89:724-730

47. Flaker GC, Blackshear JL, McBride R, et al: Antiarrhythmic drug therapy and cardiac mortality in atrial fibrillation. J Am Coll Cardiol 1992; 20:527-532 